\title{
Intestinal hymphangieclesia in an adult presented with intestinal obstruction which required laparotomy
}

Rohana AMV, Perera $\mathrm{R}$

\begin{abstract}
Intestinal hymphangieclesia has been recorded in children, often in neonates usually with other congenital abnormalities But this condition has been increasingly common in adult.
\end{abstract}

We present 60 year old patient who presented with intestinal hymphangieclesia with features of Intestinal obstruction and required laparotomy.

\section{Introduction}

Intestinal lymphangiectasia in the adult may be characterized as a disorder with dilated intestinal lacteals causing loss of lymph into the lumen of the small intestine. This results in hypoproteinemia, hypogammaglobulinemia, and lymphopenia. Most often, intestinal lymphangiectasia has been recorded in children, often in neonates, usually with other congenital abnormalities (1).

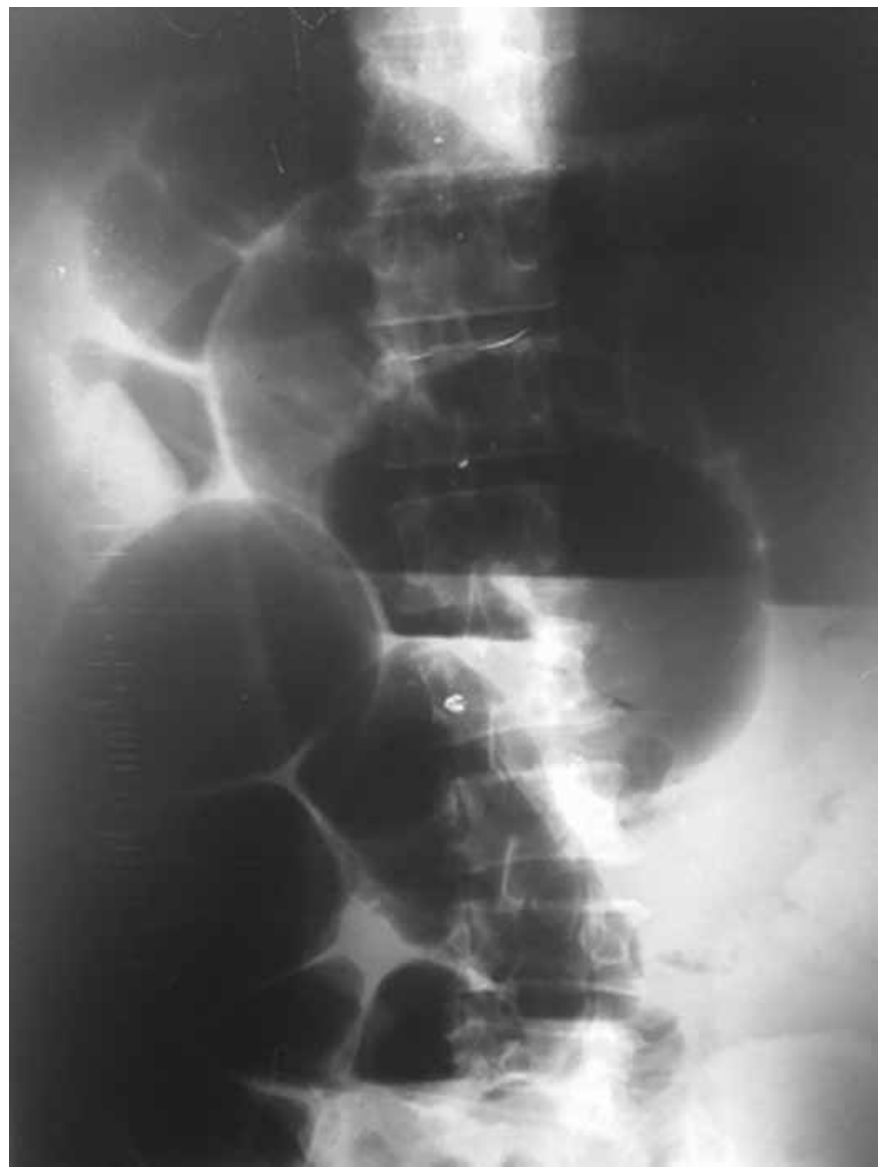

General Surgical Unit, District General Hospital Matara

\section{Case Report}

A 60 year old man presented in November 2011 with colicky abdominal pain, abdominal distension and constipation of three days. He had chronic loss of weight. Past medical and surgical history was uneventful. No past history of tuberculosis. Patient was pale, emaciated and ill looking. He did not have any lymphadenopathy. His abdomen was grossly distended. Rectum was empty. He had tachycardia and blood pressure was normal. Rest of the systems did not reveal any clinical abnormality. $X$ ray erect abdomen showed multiple fluid levels with dilated bowel loops. $\mathrm{Hb}$ was $8.9 \mathrm{~g} / \mathrm{dl}, \mathrm{WBC}$, platelets, blood urea and serum electrolytes were normal. Chest $\mathrm{x}$ ray was normal.

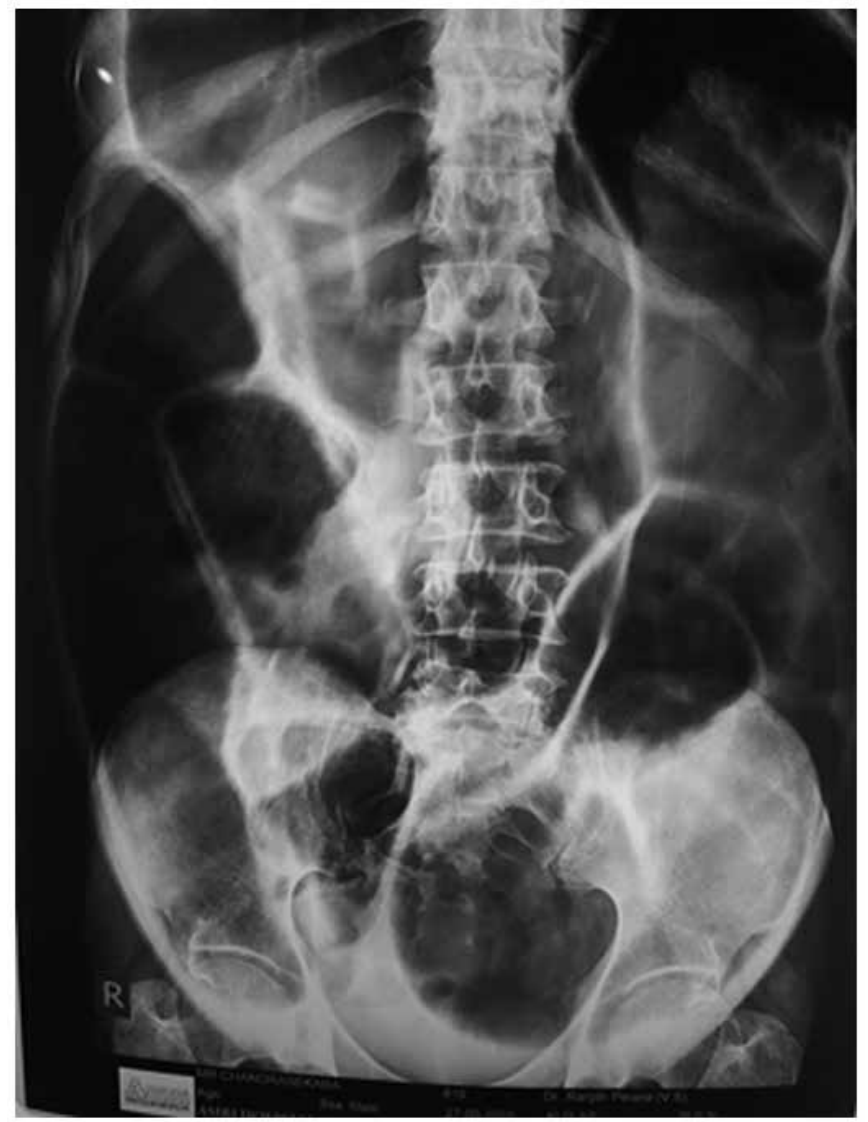

Diagnosis of small intestinal obstruction was made and an exploratory laparotomy was performed. Small intestine was grossly distended. Bowel wall was thickened and contained lot of multiple bubby cystic appearance which was extending from bowel wall to the mesentery. A part of small bowel had undergone volvulus. Obstructed part of small bowel was resected and sent for histology. End to end anastomosis was performed. Biopsy of 
small bowel revealed lymphangiectasia. The patient had an uneventful recovery However he had recurrent presentations with abdominal pain, loose motions and abdominal distension. Subsequent $\mathrm{U} / \mathrm{S}$ scan and CT scan did not show any primary cause.

\section{Discussion}

Intestinal lymphangiectasia in the adult may be characterized as a disorder with dilated intestinal lacteals causing loss of lymph in to the lumen of the small intestine and resultant hypoproteinemia, hypogammaglobulinemia, hypoalbuminemia and lymphopenia. Most often, intestinal lymphangiectasia has been recorded in children, often in neonates, usually with other congenital abnormalities but this condition in adults has become increasingly more common.

PIL is generally diagnosed before 3 years of age but may be diagnosed in older patients. Prevalence is unknown. The main symptom is predominantly bilateral lower limb edema. Edema may be moderate to severe and includes pleural effusion, pericarditis or chylous ascites. Fatigue, abdominal pain, weight loss, inability to gain weight, moderate diarrhea or fat-soluble vitamin deficiencies due to malabsorption may also be present. In some patients, limb lymph edema is associated with PIL and is difficult to distinguish lymph edema from edema (2).

Some have iron deficiency anemia, sometimes associated with occult small intestinal bleeding. Most lymphangiectasia in adults detected in recent years, however, appears to have few or no clinical featured of malabsorption. The role of surgery in intestinal lymphangiectasia is not very evident.

A case has been reported as an acute surgical emergency with pain and a mass in the right iliac fossa, which was successfully treated by resection of the involved segment of ileum 3 . Anothera case of primary intestinal lymphangiectasia, of the diffuse type, presenting with abdominal pain and voluminous diarrhea in a previously healthy 8 year - old boy has been reported. He was managed by segmental bowel resections and end-to end anastomoses. The histopathologic condition of the resected small intestine showed lymphatic dilation limited mainly to the subserosa and mesentery.. Abdominal pain and diarrhea subsided postoperatively. This case is the fourth report describing a response to operate resection (4).

A 26 - year- old patient presented with epigastric pain of sudden onset and severe puffy swelling of both legs and forearms. An irregularly shaped nodular filling defect on selective jejuna films, severe hypoproteinemia, low IgG concentration, and lymphopenia were suggestive of primary intestinal lymphangiectasia. Some weeks he developed obstructive ileus of the small intestine. On laparotomy yellowish to whitish deposits were found to be present in some segment of the small intestine and a first-sized mass $100 \mathrm{~cm}$ to the duodenojejunal flexure was resected without complications(5).

Most lymphangiectasia in adults detected in recent years, however, appears to have few or no clinical features of malabsorption. Diagnosis remains dependent on endoscopic changes confirmed by small bowel biopsy showing histological evidence of intestinal lymphangiectasia. In some, video capsule endoscopy and enteroscopy have revealed more extensive changes along the length of the small intestine. A critical diagnostic element in adults with lymphagiectasia is the exclusion of entities (e.g. malignancies including lymphoma) that might lead to obstruction of the lymphatic system. In addition, occult infections (e.g. Whipple's diseases from Tropheryma whipplei) or inflammatory disorders (e.g. Crohn's disease) may also present with profound changes in intestinal permeability and protein - losing enteropathy that also been described even decades following initial diagnosis of intestinal lymphangiectasia.

Treatment has been historically defined to include a low fat diet with medium - chain triglyceride supplementation that leads to portal venous rather that lacteal uptake. A number of other pharmacological measures have been reported or proposed but these are largely anecdotal. Finally, rare reports of localized surgical resection of involved areas of small intestine have been described but follow-up in these cases is often limited,

\section{References}

1. Freeman HJ1, Nimmo M., World J Gastrointest Oncol. 2011 Feb 15; 3 (2) : 19-23. doi: , 10. 4251/ wjgo.v3.i2.19, Intestinal lymphagiectasia in adults.

2. Orpahnet J Tare Dis. 2008, 3:5. , Published online 2008 Fed 22. doi: 10.1186/1750-1172-3-5, Primary intestinal lymphangiectasia (Waldmann's disease) Stephane Vignes ${ }^{1}$ and Jerome Bellanger ${ }^{2}$,

3. Hisham Nazer,; Chief Editor : Julian Katz, MD

4. J Pediatr Surg.2009;44(10):e13-7 primary intestinal lymphangietasia successfully treated by segment resections of small bowel, Kim NR; Lee SK; Suh YL

5. J pediatr Gastroenterol Nutr. 2001 oct; 33(4): 508-10, Primary intestinal lymphagiectasia: a rare disease in the differential diagnosis of acute abdomen. Upgualp S(1), mutus M, Kutlu O, Cetin S, Baysal, T, Mizrak B. 